\title{
A STUDY OF SENTINEL LYMPH NODE BIOPSY WITH METHYLENE BLUE DYE IN EARLY CARCINOMA OF BREAST
}

\author{
Hrishikesh Deka1, Suresh Jaywant Bhosale², Karan Kumar 3 , Yash Pandey4 ${ }^{4}$, Sourav Baur 5 , Gayatri6, Ajinkya Puppal7, Rajath Rakshit ${ }^{8}$
}

${ }_{1}^{1}$ Postgraduate Student, Department of General Surgery, Krishna Institute of Medical Sciences, Karad.

2Professor, Department of General Surgery, Krishna Institute of Medical Sciences, Karad.

${ }^{3}$ Postgraduate Student, Department of General Surgery, Krishna Institute of Medical Sciences, Karad.

${ }^{4}$ Postgraduate Student, Department of General Surgery, Krishna Institute of Medical Sciences, Karad.

${ }^{5}$ Postgraduate Student, Department of General Surgery, Krishna Institute of Medical Sciences, Karad.

6Postgraduate Student, Department of General Surgery, Krishna Institute of Medical Sciences, Karad.

7 Postgraduate Student, Department of General Surgery, Krishna Institute of Medical Sciences, Karad.

${ }^{8}$ Postgraduate Student, Department of General Surgery, Krishna Institute of Medical Sciences, Karad.

\begin{abstract}
BACKGROUND

Axillary lymph node status is very important prognostic factor in breast carcinoma patients; nodal evaluation is therefore a critical part of breast cancer management. However, axillary lymph node dissection results in significant morbidity. Thus, in the midnineties Sentinel Lymph Node Biopsy (SLNB) was introduced for staging in breast cancer patients. We carried out a study to find out whether the sentinel lymph node initially receives malignant cells from a breast carcinoma and whether a clear sentinel node reliably forecasts a disease-free axilla.
\end{abstract}

\section{MATERIALS AND METHODS}

Here 68 female patients with pathologically (FNAC/Trucut biopsy) proved early breast cancer without clinically palpable nodes, clinical stage T1/T2 N0M0, admitted in Krishna Institute of Medical Sciences, Karad from October 2014 to June 2016 who were to undergo modified radical mastectomy were selected for this study. Methylene blue dye ( $5 \mathrm{cc}$ of $1 \%$ dye) was injected in subareolar region 20 minutes prior to surgery. The breast was then massaged for 5 minutes. Intraoperatively, sentinel lymph node were searched after raising superior flap. Dissection of axillary tissue to identify stained lymph node was done. All blue nodes and any node receiving a blue lymphatic channel were considered as sentinel nodes. After excising the stained lymph nodes, complete axillary clearance and removal of breast tissue was done.

\section{RESULTS}

Sentinel Lymph Node was identified in 61 cases out of a total of 68 cases in which the procedure was performed with an identification rate of $89 \%$. The overall sensitivity, specificity, Positive Predictive Value (PPV) and negative predictive value (NPV) of SLNB in predicting axillary node status was $93.33 \%, 83.87 \%, 84.84 \%$ and $92.85 \%$ respectively. A false negative rate of $6.67 \%$ was observed.

\section{CONCLUSION}

Our results show that this simplified technique of identifying the sentinel node(s) by using subareolar methylene blue has sufficiently high identification rate (89\%). The results of the present study describe our initial experience with the SLNB technique, hence a false negative rate of $6.67 \%$ and negative predictive value of $92.85 \%$, both of which are modifiable variables.

\section{KEYWORDS}

Sentinel Lymph Node, Breast Biopsy, Methylene Blue Dye, Early Carcinoma of Breast, Lymphatic Mapping.

HOW TO CITE THIS ARTICLE: Deka H, Bhosale SJ, Kumar K, et al. A study of sentinel lymph node biopsy with methylene blue dye in early carcinoma of breast. J. Evolution Med. Dent. Sci. 2017;6(21):1701-1704, DOI: 10.14260/Jemds/2017/374

BACKGROUND
Carcinoma breast is one of the most common carcinoma occurring in females and it is a devastating illness, both physically and mentally. In 2010, a total of 209,060 cases of invasive breast cancer and almost 54,010 cases of in situ breast carcinoma were diagnosed in the United States.

Financial or Other, Competing Interest: None.

Submission 12-01-2017, Peer Review 24-02-2017,

Acceptance 03-03-2017, Published 13-03-2017.

Corresponding Author:

Dr. Hrishikesh Deka,

C/o. D. K. Deka,

House Number 4, Kanaklata Path,

Bhangagarh, G. S. Road,

Guwahati-781005, Assam.

E-mail:dr.hrishikeshdeka@gmail.com

DOI: $10.14260 /$ jemds $/ 2017 / 374$
Breast carcinoma is the leading malignancy with more than 1 million cases of breast carcinoma diagnosed worldwide each year. In India, there is an annual incidence of 75,000 cases each year. Breast cancer causes 5,19,000 deaths in a year worldwide, about 9,00,000 women are diagnosed each year. Incidence of breast cancer is $0.26 / 1,00,000$ in males and $20.1 / 1,00,000$ in females; while mortality associated with breast cancer is $1.20 / 1,00,000$ in males and $4.32 / 1,00,000$ in females. ${ }^{1}$ The incidence of breast cancer in India is on the rise and is rapidly becoming the number one cancer in females pushing cervical cancer to the second spot. It is reported that 1 in 22 women in India is likely to suffer from breast cancer during their lifetime. The current treatment of breast cancer is guided by pathology, staging and recent I nsights into breast carcinoma biology. 
Surgical treatment of breast carcinoma has evolved from Halsted's radical mastectomy to breast-conserving surgery. Similarly, surgical management of regional lymph nodes has become less extensive. It is well established that axillary lymph node status is a very important prognostic factor in breast carcinoma patients; nodal evaluation is therefore a critical part of breast cancer management. Furthermore, axillary nodal metastasis is an important parameter that indicates the need for adjuvant regional and systemic treatment. Axillary lymph node dissection also helps with the locoregional control of breast carcinoma.

However, axillary lymph node dissection results in significant morbidity. The immediate problems include acute pain and paraesthesia, need for hospitalisation, reduced range of shoulder movements and need for a surgical drain in the surgical bed for a period of 2 weeks or more. Long term problems resulting from ALND include lymphoedema of the ipsilateral arm, numbness, chronic pain and reduced range of motion at the shoulder joint.

In the mid-nineties, Sentinel Lymph Node Biopsy (SLNB) was introduced for staging in breast cancer patients. In 1959, Gould presented his results of SLNB in patients treated for cancer of the parotids followed by a report describing the retrieval of sentinel lymph nodes in penile cancer. Two decades later, Morton popularised the use of SLNB in melanoma patients. In 1994, it was Giuliano working in the same Institute as Morton, who first described the use of the procedure in breast cancer patients.

The technique of sentinel lymph node dissection was developed to reduce the morbidity associated with axillary surgery, while still providing accurate staging information. Because many patients now present with clinically nodenegative disease, SLNB can identify those with proven nodepositive disease who may benefit from complete axillary dissection.

\section{MATERIALS AND METHODS}

This was a prospective study conducted in the Department of General Surgery at Krishna Institute of Medical Sciences, Karad. Here 68 female patients with pathologically (FNAC/Trucut biopsy) proved early breast cancer without clinically palpable nodes, clinical stage T1/T2 N0M0, admitted in Krishna Institute of Medical Sciences, Karad from October 2014 to June 2016 who were to undergo modified radical mastectomy were selected for this study. Pregnant/lactating females, patients with previous breast surgery, patients with previous chemotherapy or radiotherapy, patients with multicentric or multifocal tumour, patients allergic to methylene blue dye or unwilling for the procedure were excluded from the study.

With all the required preoperative investigations, informed consent from all participating patients and after ensuring fitness for surgery, these patients were taken for SLNB along with modified radical mastectomy.

Methylene blue dye (5 cc of $1 \%$ dye) was injected in subareolar region 20 minutes prior to surgery. The breast was then massaged for 5 minutes. Intraoperatively, sentinel lymph nodes were searched after raising superior flap. Dissection of axillary tissue to identify stained lymph node was done. All blue nodes and any node receiving a blue lymphatic channel were considered as sentinel nodes. After excising the stained lymph nodes, complete axillary clearance and removal of breast tissue was done.

Lymph nodes were divided into two groups: the dye stained lymph node as sentinel node and the rest of the lymph nodes removed by axillary clearance. These along with the breast specimen were subjected to histopathological examination and pathological data including histopathological type, size of the tumour, lymph node status in sentinel and non-sentinel lymph nodes was collected.

Diagnostic Validity Tests calculating sensitivity, specificity, false negative, positive predictive value, negative predictive value and accuracy of the sentinel lymph node biopsy were done after final histopathology report was available in comparison with rest of the axillary lymph nodal status to assess the efficacy of sentinel lymph node biopsy in detecting axillary metastasis.

\section{RESULTS}

Of all the 68 patients who underwent modified radical mastectomy with axillary clearance, histological examination revealed $82.35 \%$ to be invasive ductal carcinoma, $11.76 \%$ to be lobular carcinoma and $5.88 \%$ to be medullary carcinoma with $66.17 \%$ tumours in T2 stage and $33.82 \%$ tumours in $\mathrm{T} 1$ stage. The most common location of the tumour was in the upper and outer quadrant of the breast (60.29\%).

Sentinel Lymph Node was identified in 61 cases out of a total of 68 cases, in which the procedure was performed with an identification rate of $89 \%$. The mean number of sentinel lymph nodes detected was 1.52 and mean number of axillary nodes dissected was 14 (range 5 - 21). Total number of cases with positive axillary nodes was 35 .

Among these 35 cases with positive axillary nodes, SLN was the only positive node for metastases in 5 cases (14.28\%), both SLN and rest of the axilla are also being positive for metastases in 28 cases $(80 \%)$ and in 2 cases (5.7\%) SLN was negative for metastases where axillary nodes were positive for metastasis. Total number of cases with negative axillary nodes was 26 . In all these 26 cases, SLN was also negative for metastases.

With above mentioned results the overall sensitivity, specificity, positive predictive value (PPV) and negative predictive value (NPV) of SLNB in predicting axillary node status was $93.33 \%, 83.87 \%, 84.84 \%$ and $92.85 \%$ respectively. A false negative rate of $6.67 \%$ was observed. The overall accuracy was $88.52 \%$.

\section{DISCUSSION}

Between October 2014 and June 2016, 68 patients underwent SLN biopsy with methylene blue dye as a part of study along with MRM. A prominent blue stained lymphatic channel connecting to one or more axillary sentinel nodes could be identified in all but 7 patients, indicating an identification rate of $89 \%$ ( 61 of 68 patients). In 2 out of 7 cases in which SLN could not be identified, axilla was positive for nodal metastasis. The inability to identify SLN in these cases could be attributed to surgeon's inexperience with SLNB, as most of these cases occurred in the initial period of study. As the study progressed, a SLN identification rate of $89.7 \%$ (61 out of 68) was achieved with a total of 93 SLNs identified in 61 cases (mean 1.52). 
Optimal SLN biopsy technique using an intradermal and/or subareolar injection of radioactive colloid and blue dye can improve SLN identification rates regardless of patient and tumour characteristics. Many previous studies have demonstrated that identification rate varies with surgical technique with the lowest identification rates in utilising blue dye alone $(65 \%-88 \%)$ and the highest rates in using either radioactive colloid alone $(81 \%-94 \%)$ or in combination with blue dye (81\% - 100\%). As mentioned earlier, sub-areolar and dermal injection with radioactive colloid in combination with blue dye provides the highest identification rate.

Optimal SLN biopsy technique using an intradermal and/or subareolar injection of radioactive colloid and blue dye can improve SLN identification rates regardless of patient and tumour characteristics. Many previous studies have demonstrated that identification rate varies with surgical technique with the lowest identification rates in utilising blue dye alone $(65 \%-88 \%)$ and the highest rates in using either radioactive colloid alone $(81 \%-94 \%)$ or in combination with blue dye $(81 \%-100 \%)$. As mentioned earlier, sub-areolar and dermal injection with radioactive colloid in combination with blue dye provides the highest identification rate.

\begin{tabular}{|c|c|c|}
\hline Authors & $\begin{array}{c}\text { Mapping } \\
\text { Method }\end{array}$ & $\begin{array}{c}\text { Identification } \\
\text { Rate \% }\end{array}$ \\
\hline Cox et al $^{2}$ & BD + COL & 94.1 \\
\hline Paganelli et al $^{3}$ & COL & 98 \\
\hline Veronesi et al $^{4}$ & COL & 99 \\
\hline Villa et al $^{5}$ & BD + COL & 98 \\
\hline Noguchi et al $^{6}$ & BD + COL & 94 \\
\hline Kern et al & BD & 97.5 \\
\hline Our Study & BD & 89 \\
\hline
\end{tabular}

* BD: blue dye, COL: radio-colloid

In present study, sub-areolar injection of methylene blue dye was used to locate the SLN in all the patients. The subareolar technique offers many advantages: it is easy, it avoids the need for image-guided injection and the transit time is also quicker than the peritumoural technique. ${ }^{8}$

Chemical agents used for lymphatic mapping fall into two categories. In the first category, there are various vital dyes that are used in SLNB, viz. lymphazurin (isosulfan) blue, patent blue, methylene blue and indigo carmine. In the second category, there are radiolabelled large molecules, the most common of which are various colloids, albumin and dextran

Methylene blue dye demonstrated poor lymphatic uptake, but recent work shows that it can be substituted for isosulfan blue. ${ }^{9,10}$ We used methylene blue dye to identify SLNs, as it is less expensive and more readily available. In our study, we found that it readily reaches SLN and stains the node deeply blue.

The median age of patients in our study group was 50 years who underwent modified radical mastectomy. Sentinel lymph nodes were identified in 61 of 68 patients with a mean number of sentinel nodes identified was 1.52 (range 1 - 3). The mean number of axillary nodes removed per case was 14 (range 5 - 21). Axillary node metastasis occurred in 35/61 (57\%) cases. The SLN correctly predicted the positive axillary node status in $28(80 \%)$ of 35 cases.

In 5 patients $(14.28 \%)$, SLN was the only site of metastatic disease. The false-negative result occurred in 2 cases (5.72\%). In 26 cases which did not have axillary node metastasis, SLN was identified and correctly predicted the negative axillary status. The overall sensitivity of procedure was $93.33 \%$ with specificity, positive predictive value and negative predictive value being $83.87 \%, 84.84 \%$ and $92.85 \%$ respectively. The procedure was found to have an accuracy of $88.52 \%$.

\begin{tabular}{|c|c|c|}
\hline Author & $\begin{array}{c}\text { Identification } \\
\text { Rate \% }\end{array}$ & $\begin{array}{c}\text { False Negative } \\
\text { Rate } \%\end{array}$ \\
\hline Pamar et al $^{11}$ & 77 & 16.6 \\
\hline${\text { Deo et } \mathrm{al}^{12}}^{12}$ & 90.4 & 08 \\
\hline Yu et al $^{13}$ & 86 & 09 \\
\hline Our Study & 89.07 & 6.67 \\
\hline
\end{tabular}

In two similar Indian series published earlier, Parmar et al ${ }^{8}$ using blue dye (isosulphan blue) alone reported an identification rate of $77 \%$, false negativity rate of $16.6 \%$ and a negative predictive value of $90.3 \%$. The other series by Deo et $\mathrm{al}^{9}$ using blue dye alone reported sensitivity, specificity and accuracy of SLNB in predicting the axillary node status being $84.2 \%, 100 \%$ and $91.3 \%$ respectively. The identification rate of our study is comparable with other similar studies with a false negative rate of $6.67 \%$, which tallies with other similar studies.

\section{CONCLUSION}

Our results show that this simplified technique of identifying the sentinel node(s) by using subareolar methylene blue has sufficiently high identification rate (89\%). The results of the present study describe our initial experience with the SLNB technique, hence a false negative rate of $6.67 \%$ and negative predictive value of $92.85 \%$, both of which are modifiable variables.

Routine application of this procedure along with completion ALND in carefully selected patients will provide an opportunity for both surgeon and pathologist to gain expertise and hence reduce the false negative rate to an acceptable minimum. We would like to continue doing SLNB as a routine procedure along with MRM until both surgeon and pathologists have gained sufficient expertise in identifying and correctly predicting SLN status, so that complete benefits of SLNB can be delivered to our patients in future.

\section{REFERENCES}

[1] http://www.indiatimes.com/womenarticlesshow/22 3923.cms.

[2] Cox CE, Pendas S, Cox JM, et al. Guidelines for sentinel node biopsy and lymphatic mapping of patients with breast cancer. Ann Surg 1998;227(5):645-53.

[3] Paganelli G, De Cicco C, Cremonesi M, et al. Optimized sentinel node scintigraphy in breast cancer. Q J Nucl Med 1998;42(1):49-53.

[4] Veronesi U, Paganelli G, Viale G, et al. Sentinel lymph node biopsy and axillary dissection in breast cancer: results in a large series. J Natl Cancer Inst 1999;91(4):368-73.

[5] Villa G, Gipponi M, Buffoni F, et al. Localisation of sentinel lymph node in breast cancer by combined lymphoscintigraphy, blue dye and intraoperative gamma probe. Tumori 2000;86(4):297-9. 
[6] Noguchi M, Motomura K, Imoto S, et al. A multicenter validation study of sentinel lymph node biopsy by the Japanese Breast Cancer Society. Breast Cancer Res Treat 2000;63(1):31-40.

[7] Kern KA. Sentinel lymph node mapping in breast cancer using subareolar injection of blue dye. J Am Coll Surg 1999;189:539-45.

[8] Jakub JW, Pendas S, Reintgen DS. Current status of sentinel lymph node mapping and biopsy: facts and controversies. The Oncologist 2003;8(1):59-68.

[9] Blessing WD, Stolier AJ, Teng SC, et al. A comparison of methylene blue and lymphazurin in breast cancer sentinel node mapping. Am J Surg 2002;184(4):341-5. https://www.ncbi.nlm.nih.gov/pubmed/?term=A+co mparison+of+methylene+blue+and+lymphazurin+in+ breast+cancer+sentinel+node+mapping.
[10] Simmons R, Thevarajah S, Brennan MB, et al. Methylene blue dye as an alternative to isosulfane blue dye for sentinel lymph node localization. Ann Surg Oncol 2003;10(3):242-7.

[11] Pramar V, Badwe R, Mittra I, et al. Sentinel node biopsy in operable breast cancer. Indian J Surg 2003;65:361-5.

[12] Deo S, Samaiya A, Jain P, et al. Sentinel lymph node assessment using intraoperative imprint cytology in breast cancer patients: results of a validation study. Asian J Surg 2004;27(4):294-8.

[13] Yu JC, Hsu GC, Liu YC, et al. Sentinel lymph node biopsy in early breast cancer in Taiwan. World J Surg 2002;26(11):1365-9.

https://www.ncbi.nlm.nih.gov/pubmed/12297929 